\title{
Cardiopulmonary resuscitation on television: are we miseducating the public?
}

\author{
Michael Colwill, ${ }^{1}$ Charlotte Somerville, ${ }^{2}$ Eric Lindberg, ${ }^{3}$ Caroline Williams, ${ }^{4}$ \\ James Bryan, ${ }^{4}$ Ted Welman ${ }^{3,5}$
}

'Department of Emergency Medicine, Epsom and St Helier University Hospital Trust, Carshalton, UK

${ }^{2}$ Department of Trauma and Orthopaedic Surgery, Heart of England NHS Foundation Trust Heartlands Hospital, Bordesley Green East, Birmingham, UK ${ }^{3}$ Departments of Surgery and Anaesthesia, Ashford and St Peter's NHS Foundation Trust, Chertsey, Surrey, UK

${ }^{4}$ Faculty of Medicine, Imperial College London, Charing Cross Hospital, London, UK ${ }^{5}$ Department of Emergency Medicine, St George's Hospital University Hospitals NHS Foundation Trust, London, UK

\section{Correspondence to}

Dr Ted Welman, St George's Hospital NHS Foundation Trust, Tooting SW17 0QT, UK; tedwelman@doctors.org.uk

Presented at the RCEM Annual Scientific Conference (Bournemouth, UK), September 2016.

Received 8 June 2017 Revised 20 September 2017 Accepted 21 September 2017 Published Online First 9 October 2017

\section{ABSTRACT \\ Background Out-of-hospital cardiac arrest survival rates in the UK are poor, and non-medically trained individuals have been identified to perform substandard cardiopulmonary resuscitation (CPR). Millions watch televised medical dramas and, for many, these comprise their only education on CPR. This study aims to investigate the quality of CPR portrayed on these programmes and whether this has an effect on public knowledge. \\ Methods Prospective observational study of 30 consecutive episodes of three popular medical dramas. Public knowledge of CPR and viewing habits were assessed with a survey of non-medically trained personnel.}

Results 90 episodes were reviewed with 39 resuscitation attempts shown. Chest compression rates varied from 60 to 204 compressions per minute with a median of 122 (95\% Cl 113 to 132). Depth varied from 1.5 to $7.5 \mathrm{~cm}$ with a median of 3 (3.15-4.31). Rate and depth were significantly different from the UK Resuscitation Council Guidelines (2010) $(p<0.05$, t-test). Survey participants ( $n=160,80 \%$ response rate) documented what they thought was the correct rate and depth of chest compressions and were scored accordingly. Those who documented watching medical dramas regularly scored significantly worse than those who watched occasionally ( $p<0.05$, Mann-Whitney test). Conclusion Televised medical dramas depict CPR inaccurately and laypersons may be less well informed about the correct technique the more they tune into these programmes. While there may be other confounding variables, given the popularity of television medical dramas, the poor depiction may be significantly contributing to poor public CPR knowledge and represent a potential new avenue of public education.

\section{INTRODUCTION}

At least 60000 out-of-hospital cardiac arrests (OHCA) occur in the UK annually ${ }^{1}$ with disappointingly poor outcomes: only $2 \%-12 \%$ of patients survive until discharge from hospital. ${ }^{1}$ Other developed countries perform better. ${ }^{2}$ It is well established that reducing the time from cardiac arrest to initiation of cardiopulmonary resuscitation (CPR) has a positive effect on overall outcome. ${ }^{3}$ If CPR is performed before the arrival of emergency medical services, the 30-day survival rate is significantly increased. ${ }^{4}$ OHCA survival, therefore, often relies on the ability of lay bystanders to perform effective CPR. ${ }^{56}$ Although the prevalence of bystander CPR is important, it must be effective to have a bearing on survival. Non-medically trained individuals have been identified to perform substandard CPR, exerting less force than required to achieve adequate cardiac output. ${ }^{7}$ Interestingly, some of the highest survival rates from witnessed OHCA have been documented in Seattle, where CPR has been taught in physical education for the past 30 years, indicating that a better bystander knowledge base has an effect on improving survival. ${ }^{6}$

The popularity of televised medical dramas has soared over recent years. It is no secret that these programmes seldom simulate reality with entertainment being a much higher priority than medical fact. We have noticed that these popular programmes often portray poor quality CPR. Considering that television has been shown to be an important medium by which non-medically trained personnel learn medical concepts, this seems like a wasted opportunity for public education. ${ }^{8}$ Previous studies have demonstrated that medical drama enthusiasts often overestimate the survival rate after CPR, probably a result of the wholly unrealistic and optimistic outcomes often portrayed. ${ }^{9}{ }^{10}$ It is therefore not unreasonable to question whether this poor portrayal of CPR has a similar effect on public knowledge.

We aimed to identify how well popular televised medical dramas depicted CPR. We then went on to investigate current public knowledge of CPR technique and whether this was influenced by the regularity of watching medical dramas.

\section{METHODS}

\section{Study design}

We viewed 30 consecutive episodes from each of three popular medical dramas: Grey's Anatomy, Casualty and Holby City between the broadcast period of October 2012-November 2013. All three programmes are current, fictional medical dramas. Grey's Anatomy is set in Seattle, Washington, USA, and Casualty and Holby City are both set in a fictional UK city.

Each episode was screened for occurrence of CPR. In order to allow comparison with previously published data, CPR was defined as 'any situation in which chest compressions were performed on a patient, a patient was said to be having an 'arrest', or an unconscious patient was defibrillated for ventricular fibrillation or ventricular tachycardia. ${ }^{8-11}$ For each incidence of CPR, we recorded sex and age of patient, cause of arrest, identity of the resuscitation provider, rate and depth of chest compressions (with one finger equating to $1.5 \mathrm{~cm}$ 
depth of compression), location of compressions on the chest and the immediate outcome. This was then compared with the UK Resuscitation Council guidelines. ${ }^{12}$

To test the validity of our results, two independent, blinded observers reviewed 10 episodes and recorded their findings. The observers were both trained and experienced in advanced life support (ALS) in resuscitation situations. Both observers documented identical findings both to each other and to the results recorded.

\section{Survey design}

A 10-question convenience survey was distributed among non-critically ill patients and their relatives during randomly selected shifts at a district general hospital in Surrey, UK between March and May 2015. Participants were asked if and where they had ever seen CPR (options included real life, advert, television programme, first aid course, other). They were then asked what they thought the correct rate and depth of chest compressions should be and the likelihood of immediate survival after CPR. Finally, participants were asked to disclose how often they watch medical dramas.

\section{Statistical analysis}

The one sample t-test was used to compare rate and depth of CPR from the medical drama and survey data with the UK Resuscitation Council Guidelines (2010). Depth and rate were scored objectively against the UK Resuscitation Council Guidelines (2010) for each episode of CPR observed on television to assess CPR quality. Scenes were given a score, for both depth and rate, from 0 to 3 depending on their accuracy with the most accurate answers scoring 3. To allow for direct comparison, the same scoring technique was applied to measuring the accuracy of public CPR knowledge from the survey data. The Mann-Whitney test was used to compare CPR quality across the different medical dramas and then according to resuscitation provider. Similarly, the accuracy of the public perception of CPR technique from the survey responses was then compared with where responders had been exposed to CPR and according to how regularly responders watched medical dramas.

\section{RESULTS}

A total of 90 episodes were reviewed over the period across the three medical dramas. Forty arrests were noted with 36 resuscitation attempts. Of these, 31 patients were adults and five were children, $81 \%$ were male and 19\% female. Resuscitation attempts performed on children were excluded. Trauma was the leading cause of cardiac arrest (28\%). Others included sepsis, haemorrhage, tension pneumothorax and electrolyte abnormalities. Every resuscitation attempt was performed by a healthcare professional, of which 64\% were doctors, 31\% were nurses and $5 \%$ by another member of the multidisciplinary team. Three CPR attempts included two or more healthcare professionals performing chest compressions resulting in a total of 39 instances of CPR displayed.

\section{Assessment of chest compressions}

The rate of chest compressions varied from 60 to 204 compressions per minute $(n=39)$ (figure 1$)$. Median rate was calculated as 122 (95\% CI 113 to 132). This was compared with the UK Resuscitation Council Guidelines (2010). We found a significant difference ( $p<0.05$, one sample t-test) between the rate observed and the national guidelines of 100-120 compressions per minute. ${ }^{12}$ Compression depth varied from 1.5 to $7.5 \mathrm{~cm}(\mathrm{n}=39)$

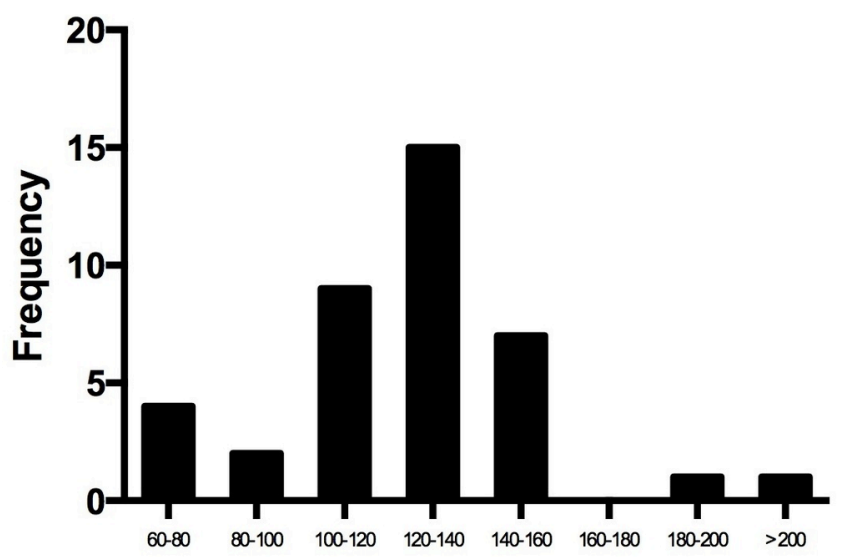

Rate of chest compressions per minute

Figure 1 Rate of chest compressions observed during cardiopulmonary resuscitation performed on the three medical dramas investigated (Grey's Anatomy, Casualty, Holby City).

(figure 2) with a median of three (95\% CI 3.15 to 4.31 ). Similarly, a significant difference was identified between the depth observed and the UK national guidelines of 5-6 cm $(p<0.0001$, one sample t-test). ${ }^{12}$ Eighty-seven per cent of resuscitators had their hands placed in the centre of the patient's chest, with the remaining $13 \%$ of the visible locations being offset to either the left or right.

Grey's anatomy resuscitations $(n=10)$ had a median rate of 97 compressions per minute (60-156) and a median depth of $2 \mathrm{~cm}(1.5-4.5)$. Casualty resuscitations $(n=13)$ had a median rate of 124 compressions per minute (96-181) and a median depth of $4.5 \mathrm{~cm}$ (3-7.5). Holby city had a median rate of 145 compressions per minute (108-204) and a median depth of $3 \mathrm{~cm}$ (1.5-4.5). Results were scored according to the distance from the guideline rate and depth to allow direct comparison. Casualty scored better than the other two programmes and was found to be significantly closer to the guidelines than Grey's Anatomy $(\mathrm{p}<0.05$, Mann-Whitney test) (figure 3).

\section{Survey responses}

A total of 160 responses were collected from the 200 surveys distributed, a response rate of $80 \%$. Thirty-two were completed by members of the healthcare profession and were therefore

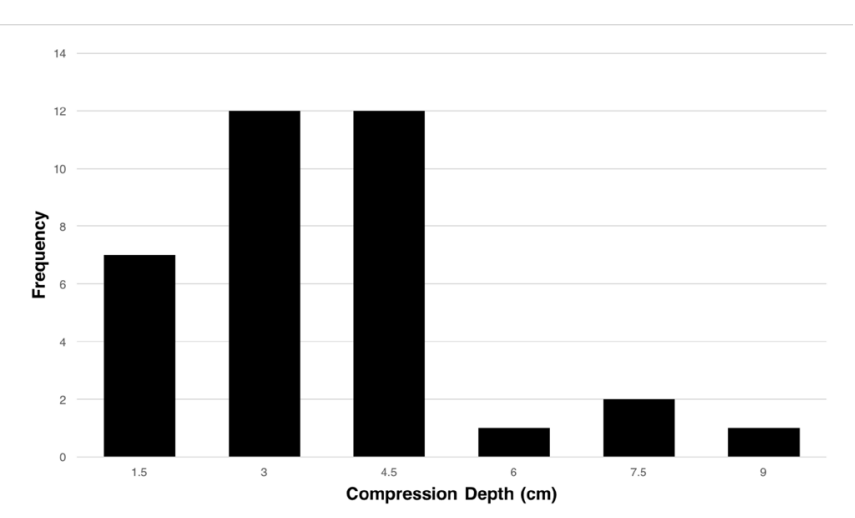

Figure 2 Depth of chest compressions observed during cardiopulmonary resuscitation performed on the three medical dramas investigated (Grey's Anatomy, Casualty, Holby City). 


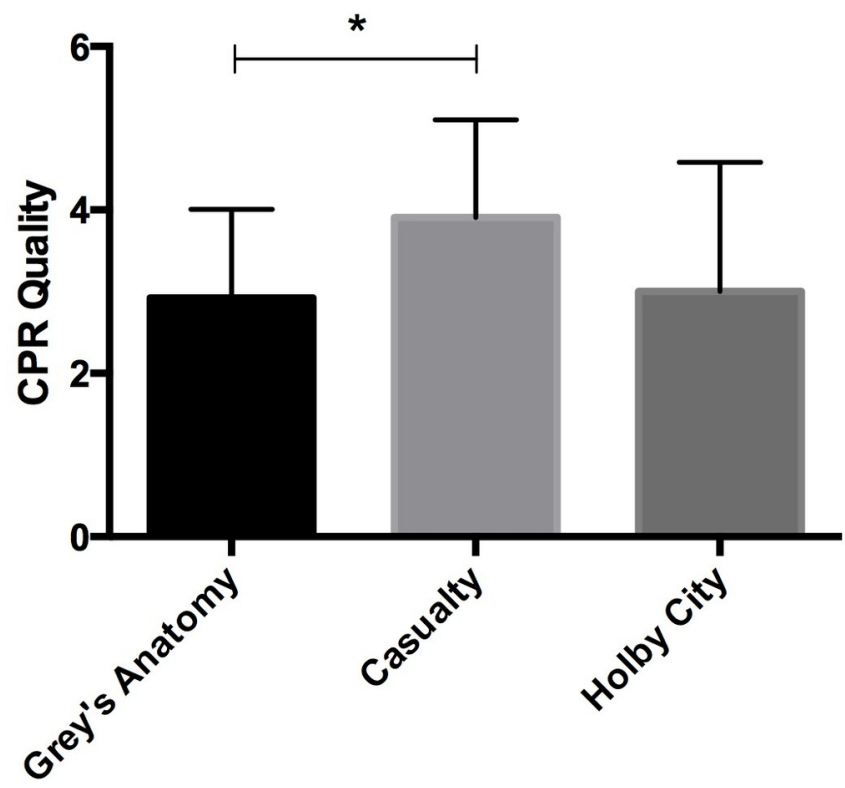

Figure 3 Cardiopulmonary resuscitation (CPR) quality by TV programme. CPR quality refers to similarity to the published guidelines in regard to compression depth and rate (three points each for correct rate and depth). CPRs performed in episodes of Casualty were significantly more accurate than those in Grey's Anatomy. Asterisk indicates statistical significance where $p<0.05$.

excluded. Of the remaining 128 responses, complete data were available from 126. The median rate of chest compressions selected was 70 compressions per minute (95\% CI 78.2 to 87.6) and median depth was $3.5 \mathrm{~cm}$ (3.29-3.84). Forty-nine responses were from those aged 16-30, 45 from those aged 31-65 and 32 from those aged over 65 . No significant difference was identified in responses between different age groups. Perceptions of how to perform CPR were compared with the UK Resuscitation Council Guidelines (2010). ${ }^{12}$ A significant difference was identified between the responses and the guidelines with regard to both depth and rate in all age groups $(p<0.0001$, one sample t-test).

Responders were asked where they had seen CPR performed. Twenty-eight people reported having only seen CPR on television and 15 had witnessed CPR firsthand. A further 28 had never seen CPR performed and 45 of the responders documented having attended a first aid course (figure 4), while the remaining participants documented a combination. Responses

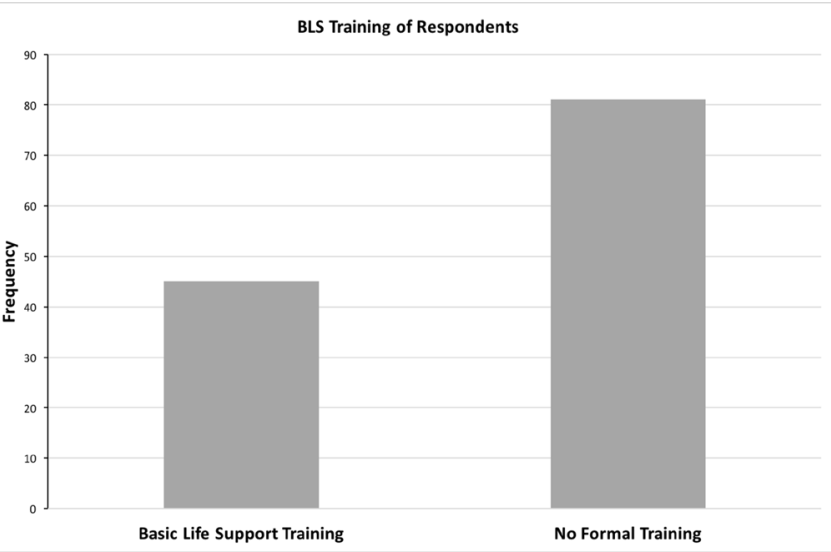

Figure 4 Prior basic life support (BLS) training in survey respondents.

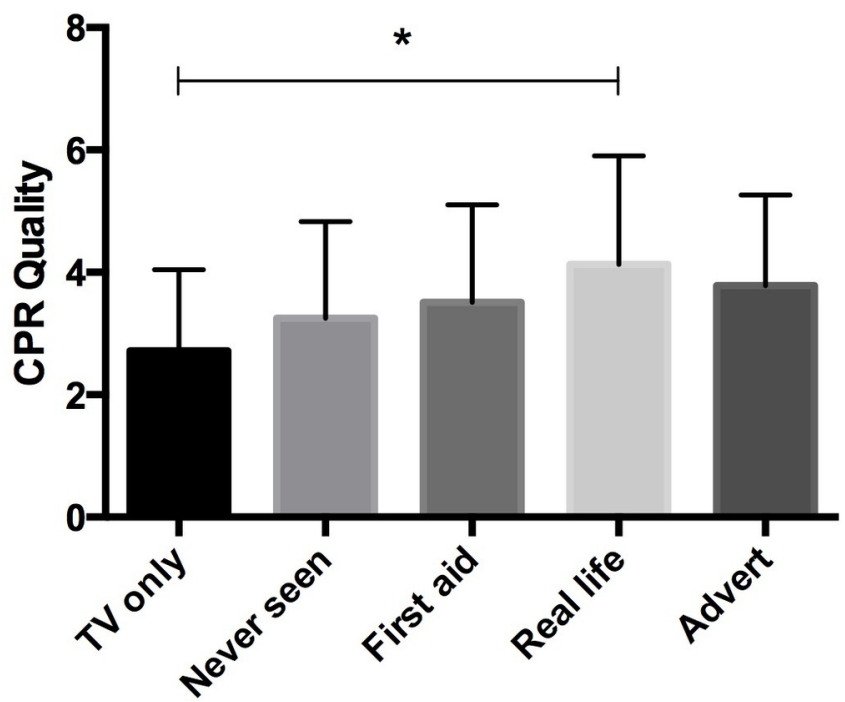

Figure 5 Knowledge of cardiopulmonary resuscitation (CPR) technique by previous responder exposure. CPR quality refers to similarity to the published guidelines in regard to compression depth and rate (three points each for correct rate and depth). Responders that had seen CPR performed in real life scored significantly better than those who had seen it on TV alone. Asterisk indicates statistical significance where $p<0.05$.

were scored with regard to their accuracy with respect to the guidelines and compared. Those who had seen CPR performed firsthand were significantly more accurate in their description of chest compressions than those who had seen it on television alone ( $<<0.05$, Mann-Whitney test) (figure 5). People who had never witnessed CPR from any form of medium did better than those who had seen it on TV alone, although this was not significant ( $p=0.14$, Mann-Whitney test). No significant difference was identified between those who had attended a first aid course and those who had only seen CPR on television or not at all ( $p=0.065, p=0.64$, respectively, Mann-Whitney test).

Survey responders were asked to divulge how often they watch televised medical dramas. Fifty-seven reported 'never', 49 reported 'occasionally (less than twice per week)' and 20 reported 'regularly (more than twice per week)'. Scores measuring the accuracy of responders' chest compression technique was then compared with how often medical dramas were viewed. A significant difference was identified between the group who watched dramas regularly and those who only watched occasionally, with regular viewers scoring significantly worse $(p<0.05$, Mann-Whitney test) than their less avid counterparts (figure 6). It should be noted that $52 \%$ of respondents who documented watching medical dramas 'occasionally' had previously attended some form of basic life support training as opposed to $38 \%$ in the group that documented 'regularly'.

\section{Survival after CPR}

Return of spontaneous circulation occurred in $62 \%$ of patients in the observed medical dramas. The quality of CPR had no bearing on the survival outcome $(p=0.59$, Mann-Whitney test).

\section{DISCUSSION}

Television is well established to be an important source of health information for members of the general public. ${ }^{13}$ We have found that CPR on televised medical dramas is inadequately performed. Both the depth and rate of chest compressions were found to be 


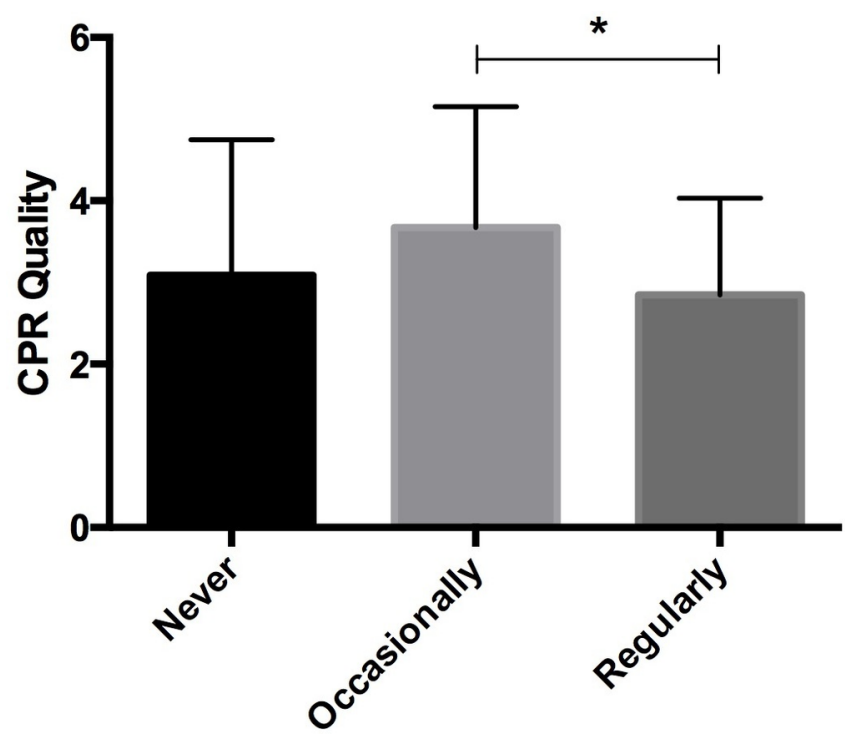

Figure 6 Public knowledge of how to perform chest compressions depending on regularity of watching medical dramas (never, occasionally (less than twice per week), regularly (more than twice per week). Cardiopulmonary resuscitation quality refers to mean accuracy of rate and depth of chest compressions recorded by survey responders (three points each for correct rate and depth). Asterisk indicates statistical significance where $\mathrm{p}<0.05$.

significantly different from the published guidelines across the episodes of resuscitation observed. Although Casualty depicted a significantly more accurate portrayal of chest compressions than Grey's Anatomy, all programmes were found to be wholly inadequate.

Televised medical dramas do employ a team of medical advisors to help write a medically accurate script. These advisors tend to be Consultant or senior nurse level. They are typically based off-site, providing telephone and email support to the show's writers and therefore do not have a significant role in critiquing the portrayal of CPR on set. ${ }^{1415}$ Perhaps, further training and a more hands on and practical approach by these trained medical advisors would result in a better depiction of CPR on television.

The results from the survey produced some interesting findings. Members of the public were scored on their knowledge of CPR and this was compared with the regularity of tuning into popular televised medical dramas. Interestingly, those that documented watching dramas more than twice per week scored significantly worse than those less committed fans that only tune in occasionally. This may indicate that the unrealistic depiction of CPR technique on television has a direct negative impact on the public's perception of how to perform this lifesaving procedure. This is concerning given that Casualty, Holby City and Grey's Anatomy received an average live audience (in the airing country) of 5.21, 4.34 and 8.96 million, respectively, during the episodes studied. ${ }^{16-21}$ These results may, however, be as a result of the discrepancy in prior first aid training of respondents, although no significant difference was identified in CPR knowledge between those who had attended formal training and those who had not.

Unsurprisingly, the knowledge of how to perform CPR was found to be relatively poor across the board, even from respondents who had been on a first aid course in the past. This might be as a result of a significant length of time having elapsed between attending the course and completing the survey; however, this information was not collected. As respondents were not healthcare professionals, they would be very unlikely to use any knowledge learnt on a first aid course on a regular basis and so would likely deskill quickly in the time following their training. The surveys showed that responders who had seen CPR on television alone scored significantly worse than those who had witnessed it exclusively in real life and those who had never witnessed it at all, although the latter was not statistically significant. While this is likely to be multifactorial in causation, the possible impact of medical drama depiction is compelling. For example, it could be that seeing CPR in real life results in a more vivid memory of the technique than watching it on TV. In addition, the CPR witnessed firsthand is likely to have been performed by a trained healthcare professional and therefore more accurate than that performed on TV.

Although not addressed in this study, it would be interesting to investigate the willingness of lay people to perform CPR and compare this with the regularity they tune into medical dramas. Could it be that those with a greater interest in medical dramas are the people that are more likely to help in a cardiac arrest situation? If this is the case, it is imperative that this group of people should be better informed on CPR technique in order to provide effective resuscitation. Equally, exposing this subset of laypersons to accurate CPR on television could be a relatively simple and targeted way of getting willing members of the public to perform better quality CPR.

The medical dramas investigated in this study are unlikely to be alone in their depiction of CPR on television. Consequently, it is not unreasonable to estimate that a substantial audience of non-medically trained personnel are being exposed to inaccurate portrayals of CPR. Given the huge viewing figures of these and other shows, improving the accuracy of medical dramas could be a relatively simple way to educate a large target population on a regular basis. This could potentially be achieved by introducing guidelines for these dramas to adhere to when filming scenes that depict CPR. In addition, it may well be beneficial to encourage producers to employ an onsite medical advisor during production to help improve CPR depiction.

There are several limitations in this study. First, there were only a relatively small number of resuscitation attempts observed across the three medical dramas. Second, all chest compressions were compared with the UK resuscitation council guidelines to allow direct comparison despite the fact that Grey's Anatomy is an American programme and would therefore be expected to adhere to the American Heart Association guidelines. This was deemed relatively insignificant in light of the similarity between the two sets of guidelines. Third, while the estimate of depth of compressions was assessed by independent and experienced ALS trained individuals, this was a complicated and difficult undertaking, confounded by the fact that many of the CPR clips were short in length.

\section{CONCLUSION}

Reducing the time to initiation of good quality CPR after cardiac arrest is essential to improving the outcome, especially in the out-of-hospital setting for which survival in the UK is poor. Our study shows that the portrayal of CPR in television dramas is inadequate. Our findings may also indicate that the individuals who watch these dramas regularly are more likely to have substandard knowledge of how to perform effective $\mathrm{CPR}$. This could indicate that this poor representation is actually miseducating lay viewers. Although a larger study that further addresses some of the confounding variables would be required, we feel that our results should promote a discussion 
on using medical dramas as an opportunity for mass public health education.

\section{Main messages}

- Cardiopulmonary resuscitation (CPR) portrayed on televised medical dramas does not adhere to published guidelines.

- Members of the public are significantly less well informed about CPR technique the more they tune into these popular programmes.

- Medical dramas are acting as a wasted opportunity to subconsciously educate the public on CPR.

\section{Current research questions}

- Could medical dramas act as a medium for mass public health education?

- Do medical dramas influence the willingness of laypeople to provide immediate cardiopulmonary resuscitation (CPR) when they witness a cardiac arrest?

- Would the quality of bystander CPR improve with better public education?

- Can health education in the media help improve survival after out-of-hospital cardiac arrest?

Contributors All authors contributed to the design of the study. EL, CS and TW selected the questions for the survey. MC, CW and JB collected the study data. EL and TW tested the validity of the results. TW wrote the first draft of the manuscript and is the guarantor for the study. All authors critically revised the final manuscript, have approved the final version and agreed to be accountable for all aspects of the work

Competing interests None declared.

Provenance and peer review Not commissioned; externally peer reviewed.

(c) Article author(s) (or their employer(s) unless otherwise stated in the text of the article) 2018. All rights reserved. No commercial use is permitted unless otherwise expressly granted.

\section{REFERENCES}

1 Perkins GD, Cooke MW. Variability in cardiac arrest survival: the NHS ambulance service quality indicators. Emerg Med J 2012;29:3-5.
2 Lindner TW, Søreide E, Nilsen OB, et al. Good outcome in every fourth resuscitation attempt is achievable - an utstein template report from the stavanger region. Resuscitation 2011;82:1508-13.

3 Hara M, Hayashi K, Hikoso S, et al. Different impacts of time from collapse to first cardiopulmonary resuscitation on outcomes after witnessed out-of-hospital cardiac arrest in adults. Circ Cardiovasc Qual Outcomes 2015:8:277-84.

4 Hasselqvist-Ax I, Riva G, Herlitz J, et al. Early cardiopulmonary resuscitation in out-ofhospital cardiac arrest. N Eng/ J Med 2015;372:2307-15.

5 Sasson C, Rogers MA, Dahl J, et al. Predictors of survival from out-of-hospital cardiac arrest: a systematic review and meta-analysis. Circ Cardiovasc Qual Outcomes 2010;3:63-81

6 Malhotra A, Rakhit R. Improving the UK's performance on survival after cardiac arrest. BMJ 2013;347:f4800

7 Geddes LA, Boland MK, Taleyarkhan PR, et al. Chest compression force of trained and untrained CPR rescuers. Cardiovasc Eng 2007;7:47-50.

8 Gordon PN, Williamson S, Lawler PG. As seen on TV: observational study of cardiopulmonary resuscitation in British television medical dramas. BMJ 1998:317:780-3

9 Van den Bulck J, Damiaans K. Cardiopulmonary resuscitation on flemish television: challenges to the television effects hypothesis. Emerg Med J 2004;21:565-7.

10 Diem SJ, Lantos JD, Tulsky JA. Cardiopulmonary resuscitation on television. miracles and misinformation. N Engl J Med 1996;334:1578-82.

11 Harris D, Willoughby $H$. Resuscitation on television: realistic or ridiculous? a quantitative observational analysis of the portrayal of cardiopulmonary resuscitation in television medical drama. Resuscitation 2009;80:1275-9.

12 Resuscitation Council (UK). Resuscitation guidelines, 2010.

13 Korownyk C, Kolber MR, McCormack J, et al. Televised medical talk shows - what they recommend and the evidence to support their recommendations: a prospective observational study. BMJ 2014;349:97346.

14 Life behind the scenes. Life as a TV medical advisor with lain Beardsell. http://blogs. bmj.com/emj/2014/03/26/life-behind-the-scenes-life-as-a-tv-medical-advisor-withiain-beardsell/ (accessed 29 Aug 2017).

15 Leaders in medical education: Grey's Anatomy medical advisor \& neurosurgeon, Dr Allan Hamilton. http://blog.osmosis.org/2014/04/24/leaders-in-medical-educationgreys-anatomy-medical-advisor-neurosurgeon-dr-allan-hamilton/ (accessed 29 Aug 2017)

16 Grey's Anatomy (season 9). https://en.wikipedia.org/wiki/Grey's_Anatomy_(season_9) (accessed 18 July 2015)

17 Grey's Anatomy (season 10). https://en.wikipedia.org/wiki/Grey's_Anatomy (season_10) (accessed 18 July 2015).

18 Casualty (series 27). https://en.wikipedia.org/wiki/Casualty_(series_27) (accessed 18 July 2015).

19 Casualty (series 28). https://en.wikipedia.org/wiki/Casualty_(series_28) (accessed 18 July 2015).

20 Holby City (series 15). https://en.wikipedia.org/wiki/Holby_City_(series_15) (accessed 18 July 2015).

21 Holby City (series 16). https://en.wikipedia.org/wiki/Holby_City_(series_16) (accessed 18 July 2015). 